\title{
La Fête interrompue dans les contes littéraires québécois du XIXe siècle
}

\author{
Pierre Rajotte \\ Centre de recherche en littérature québécoise, \\ Université Laval, Québec
}

\section{$\mathrm{L}$}

'imaginaire populaire dépend largement de ce que Nietzsche appelle "l'esprit dionysiaque." Dans sa pulsion primordiale, il revêt un caractère d'abord carnavalesque, pour employer l'expression de Mikhaïl Bakhtine (1970), c'est-à-dire orienté vers la fête et le désordre. C'est pourquơ 11 a rarement droit de cité en littérature, à moins d'être récupéré à d'autres fins, généralement répressives. Le plus souvent, en effet, la littérature, apanage d'une élite en rapport de supériorité avec la masse, exerce d'une façon ou d'une autre une violence symbolique sur le peuple pour rétablir l'ordre menacé. La part libératrice et subversive de l'imagination populaire est alors édulcorée sinon occultée au profit de préceptes qui raffermissent l'ordre établi.

Cette affirmation est particulièrement vraie au XIXe siècle de la littérature canadienne qui s'est formée en interaction avec la tradition orale, mais aussi et surtout en réaction contre elle. Dans le but de désamorcer les débordements carnavalesques trop souvent en rupture avec la morale officielle, les lettrés ont opéré dans les croyances populaires une sélection en fonction de valeurs purement morales. Afin de l'illustrer, nous nous sommes intéressé au thème de la fête populaire dans les contes littéraires québécois du XIXe siècle. Dans un pays et à une époque où existe un penchant très marqué pour le fête, occasion par excellence de défoulement et de libération des tabous, il y a lieu de s'interroger sur le sens que lui ont donné les écrivains. Dans sa "Lettre à d'Alembert sur les spectacles," Jean-Jacques Rousseau opposait la fête qui recompose de nouveaux rapports humains et fonde le "contrat social" en dehors des institutions établies, et le thêâtre inséparable d'une élite dominante qui, pour ses spectacles, isole un individu afin de le détruire par la règle ou la dérision. Il résumait bien alors tout l'aspect contradictoire de la fête, qui, selon qu'elle est l'occasion d'encourager ou de dénoncer les conduites déviantes, renouvelle ou conforte la structuration sociale. Selon Gilbert Durand (1969, p. 358-359), "la fête est à la fols moment négatif où 
les normes sont abolies, mais aussi joyeuse promesse à venir de l'ordre ressuscité." Qu'en est-il dans les contes? La fête suggère-t-elle dans une intuition momentanée l'image d'une communauté différente de celle de la société dans laquelle elle se manifeste, ou est-elle plutôt réduite à un rituel victimaire pour préserver l'ordre établi? Jusqu'à quel point la fête évoque-t-elle le "retour au chaos d'où doit sortir l'être régénéréy pour reprendre l'expression de Durand (1969, p. 358)? Voilà autant de questions auxquelles nous avons tenté de répondre en tenant compte du rapport dialogique qui s'instaure entre le projet d'une littérature nationale au Québec au XIXe siècle et la récupération de la tradition orale qu'il sous-tend.

La démesure d'un continent neuf à habiter, le climat rigoureux à maîtriser, de même que l'analphabétisme d'un peuple préoccupé par sa survivance, tendent à perpétuer en Nouvelle-France les formes primitives de l'esprit carnavalesque, caractérisé par la transgression des interdits dans l'excès dionysiaque du boire, du manger, de la sexualité, du rire et de la violence. Selon les témoignages de plusieurs historiens et voyageurs de l'époque, le peuple canadien-français se distingue par son penchant marqué pour la fête. Dès le début de la colonie, Champlain fonde l'Ordre du Bon Temps, une société de convivialité, afin de lutter contre les durs hivers du Canada. Plus tard, surtout à la campagne, les moeurs ont une liberté que même les auteurs les plus circonspects reconnaissent. Joseph-Charles Taché (1863, p. 62) en témoigne de façon fort êloquente:

C'était dans le temps des bonnes années [...]. J'ai connu des habitants quil achetaient une tonne de rhum et un baril de vin pour leur provision de l'année; la carafe et les verres avec les croxignoles étalent toujours sur la table, tout le monde était invité, on ne pouvait pas entrer dans une maison sans prendre un coup. [...] Les fêtes étaient presque continuelles, il n'y avait pour ainsi dire, que dans les saisons des semences et des récoltes que l'on travaillait. On avait même fait un refrain, que le maitre de la maison chantait dès que ses visiteurs faisaient mine de partir: "Les Canadiens sont pas des fous: Partiront pas sans prendre un coup!" 
Jusqu'en 1840 , le clergé, encore peu nombreux au pays, demeure impuissant devant de tels abus. Certes, il tend à moraliser avant même que les réjouissances commencent, mais il a beau dire et beau faire, la fête lui échappe aussitôt qu'elle est sortie de l'église. «Toutes les sources, note Jean-Pierre Wallot (1973, p. 203-205), se recoupent pour nous camper une masse indocile, attachée aux plaisirs de la vie et souvent corrompue." Le renouveau religieux des années 1840 marque cependant un point tournant. Profitant de la désillusion politique causée par la défaite des patriotes, le clergé lance une entreprise concertée visant à transformer les attitudes et les conduites religieuses de la masse des fidèles. Dès lors, les moyens de ramener les fêtards à l'ordre se multiplient. Les prédications célèbres de Mgr de Forbin-Janson et les campagnes de tempérance de l'abbé Chiniquy inaugurent un véritable modèle de reconquête pastorale. Tels de nouveaux Jonas menaçant Ninive des foudres célestes, ils appellent les fidèles à résipiscence et les embrigadent massivement dans le mouvement de Tempérance qui constitue une forme de régulation efficace de la déviance décrite comme la plus menaçante. De plus, pour faire pièce à la fête populaire, on ne tarde pas à lui substituer la fête officielle, occasion de célébrer l'ordre établi par d'importantes processions religieuses (de la Fête-Dieu, du Sacré-Coeur, de Sainte-Anne). Enfin, on s'attaque à l'imaginaire, comme l'illustre un ambitieux projet de littérature nationale voué à la récupération de la tradition orale.

Il va sans dire que, chez un peuple encore en majorité analphabète, la tradition orale jouit d'une prédominance incontestée. Consciente de ce fait, l'élite entend en tirer profit. Pendant la décennie de 1860 en particulier, les promoteurs de la littérature nationale valorisent tout genre provenant du peuple comme en témoigne la fondation de la revue littéraire Les Soirées canadiennes et l'épigraphe inspiré de Charles Nodier dont elle se réclame: "Hâtons-nous de raconter les délicieuses histoires du peuple avant qu'il les ait oubliées." Selon Joseph-Charles Taché (1863. p. 17), les légendes et les contes «dans lesquels les peuples ont versé leur âme, avec lesquels ils ont cherché à satisfaire, dans certaines limites, ce besoin de merveilleux qui est le fond de notre nature [...], constituent une portion notable, le fonds on peut dire, de toute littérature nationale." D'inspiration romantique, cette conception de la littérature présuppose que c'est d'abord par son imaginaire qu'un peuple exprime son génie propre, imaginaire certes des grands artistes et des grands écrivains, mais aussi imaginaire collectif, peut-être moins délicat, mais plus authentique et sûrement plus prometteur d'originalité. A ce titre, les Canadiens, bien qu'encore sans littérature et sans beaux-arts, jouissent de l'avantage de 
pouvoir compter sur une tradition orale extrêmement riche. Aussi est-ce une vaste entreprise de récupération du patrimoine folklorique que proposent les collaborateurs des Soirées canadiennes.

Mais jusqu'à quel point la tradition orale ainsi récupérée présente-t-elle au peuple sa propre image? Les écrivains doivent-ils se limiter à un simple rôle de transcripteurs? La réponse ne tarde pas à venir. Dès le premier numéro de la revue, certains collaborateurs indiquent bien l'orientation qu'ils entendent donner à l'entreprise. Aux yeux du docteur Hubert Larue (1861, p. 145), pour ne mentionner que celui-là, l'intérêt de "ces légendes naĩves" réside essentiellement dans le fait qu'elles "peignent si bien la bonne foi de nos ancêtres, leur esprit si éminemment religieux, en même temps qu'elles rappellent leur noble origine." Tacitement, on procède donc à une réinterprétation du folklore. Tout en prétendant le préserver, on le modifie en fonction de son idéologie de classe.

Le répertoire du conteur populaire au Québec, note Maurice Lemire (1993), comporte deux volets non nécessairement étrangers l'un à l'autre. Le premier narre les exploits et les prouesses plus ou moins véridiques accomplis par les voyageurs des pays d'en haut. En fait, dans une société où prime le travail manuel, il n'est pas êtonnant qu'une bonne partie de l'imaginaire collectif se développe autour des rêveries de force et de résistance physique. Mais ce n'est pas vraiment de ce côté que les littéraires cherchent leur inspiration. L'exaltation de la force physique qui fait de certains dévoyés des sortes d'idoles contient trop de germes d'anarchie. Il vaut mieux se tourner du côté du fantastique, dans lequel l'intervention des êtres surnaturels apparait comme une prolongation des forces archétypales du bien et du mal. Compte tenu des objectifs qu'on poursuit, ce second volet du répertoire des conteurs populaires présente en effet des avantages indéniables. Centré sur le diable et ses avatars, tels que loups-garous, feux follets, revenants, il favorise le prolongement de la religion populaire selon laquelle le bien est toujours récompensé et le mal, puni. A ce titre, il revête un caractère nettement moralisateur.

Dans ce contexte, la fête prend un sens tout à fait particulier. La stratégie la plus commune consiste à organiser un contre-discours pour condamner les abus qu'elle engendre. Or c'est dans ce contre-discours que nous trouvons peut-être le meilleur témoignage sur les ravages de livrognerle et de la libation dans les campagnes. Dans le conte "Le Feu des Roussi» de Faucher de Saint-Maurice (1872, p. 82), la vigueur avec laquelle "Marie la couturière" sermonne Cyprien "le roi des noceurs" prouve à quel point les méfaits de l'alcoolisme inquiétaient l'élite de la 
société: "Ah! Cyprien, lui dit-elle, il est pénible de vous voir, vous fils d'habitant, boire votre champ, au lieu de le cultiver."

Ainsi, dans un imaginaire revu et corrigé par la classe dominante, la fête n'éclate que pour rendre plus manifeste le désordre à réprimer. Que ce soit par larrivée d'un invité inattendu, le plus souvent le diable ou un revenant, par le malaise d'un convive qui se transforme en loup-garou, ou par la voix d'un conteur, elle est toujours interrompue au profit du rétablissement de l'ordre.

\section{La Narration d'un récit}

L'événement particulier que représente la narration d'un récit a souvent pour effet de mettre fin à la fête. Dans la description qu'il fait de la fête de la Grosse Gerbe, Édouard Duquet (1866, p. 25), notamment, précise que: "Les chants, les jeux et les danses étaient suivis d'histoire de loups-garous, de fantômes et de revenants." L'effet terrifiant de ces histoires (ou récits seconds) ne manque jamais de refroidir l'ardeur des fêtards (du récit premier) comme en témoigne la réaction des invités du père Joseph Hervieux, dans un conte de Honoré Beaugrand (1979, p. 91-92). Après avoir écouté la légende du "fantôme de l'avare," ceux-ci n'ont plus le coeur à la fête. L'un des convives, le père Montépel, offre "une dernière rasade de bonne eau-de-vie de la Jamaïque" qu'on but, de dire le narrateur, "avec moins d'entrain que les autres, car l'histoire du maittre d'école [le fantôme de l'avare] avait touché la corde sensible dans le coeur du paysan franco-canadien: la croyance à tout ce qui touche aux histoires surnaturelles et aux revenants." Puis "après avoir salué cordialement le maitre et la maitresse de céans et s'être redit mutuellement de sympathiques bonsoirs, garçons et filles reprirent le chemin du logis."

La narration d'un conte interrompt la fête parce qu'elle constitue elle-même une fête. Comme le dit si bien François Ricard (Honoré Beaugrand, 1979, p. 6), le conteur convie toujours ceux quil l'écoutent à kune rupture dans le cours régulier de la vie et à l'entrée dans un ordre tout à fait différent." Comme au thêâtre, quand résonnent les trois coups, le spectateur s'apprête à passer d'un monde dans un autre. C'est aussi ce que font les auditeurs du conte lorsque le diseur annonce rituellement qu'il va les émerveiller: «Pour lors que je vais vous raconter une rôdeuse d'histoire ..." (Honoré Beaugrand, 1979, p. 17). A elle seule, cette phrase suffit à provoquer un changement dans la vie des auditeurs. Aussitôt tout se tait, tout le visible s'évanouit, et chacun consent d'avance à la merveille. La narration du conte 
devient alors un acte cathartique de compensation, un exutoire à tous les désirs inassouvis. Elle donne accès à un monde compensatoire, imaginaire, celui du désir où plus aucun interdit n'a force de loi. Dans cet intervalle peuvent se réaliser les rêves les plus fous, se commettre les méfaits qui dispensent d'avoir à les perpétrer soi-même, mais surtout dissuade de le faire car dans les contes québécois "toute transgression entraîne obligatoirement une punition, toute faute nécessite, à coup sûr, réparation" (Aurélien Boivin, 1987, p. 11).

La légende "Le Rigodon du diable" de Louvigny de Montigny (1898, p. 5), illustre on ne peut mieux ce rituel cathartique et répressif qui fonde le retour à l'ordre et la détente de la vie courante. Après la procession du Mardi gras, l'enterrement du carnaval, qui marque le passage abrupte de la période des réjouissances à la période des pénitences (le carême), s'accomplissait sous les toits dans le village de SainteAgathe-des-Monts. "On fêtait partout, et particulièrement chez le maire Lafantaisie." "On s'amusait ferme" de dire l'auteur:

Après éputsement du répertoire chorégraphique, on avait improvisé un reel [danse populaire] dont l'exécution promettait de faire crouler la maison sur l'enthousiasme des danseurs. Le beau Chartrand, avec son crincrin, se démenait comme un épileptique [...] le petit Fournier, à qui ses souliers neufs pesaient, venait de les enlever et se reprenait à sauter avec un regain d'entrain; le gros Latour, qui suait comme une outre, avait jeté sa bougrine; enfin Poméla $[\ldots]$ n'en était ni plus ni moins qu'au délire ...

Soudain, à minuit, comme le veut la coutume, le père Lafantaisie intervient pour interdire la danse "sur le mercredi des cendres," c'est-à-dire pendant le carême. Devant l'insistance de ses invitês, il décide alors de leur raconter une histoire pour leur faire entendre raison. Subitement la fête prend une tournure nettement plus ordonnée.

"W'ai justement un fion d'histoire qui va vous montrer pourquoi j'veux pas vous laisser danser. T'nez, pas de blagues, chaque fois que j'y pense, j'en tremble des pieds à la tête, et, quand j'y r'pense, j'en tremble de la tête aux pieds." A ce prélude apocalyptique, la grosse Poméla se rapproche de sa mère, par peur de rester près de 
la porte d'entrée; le petit Fournier rechausse ses souliers; chacun se met d'aplomb sur sa chaise ...

Le père Lafantaisie raconte alors que trente ans auparavant, alors qu'il regagnait la demeure paternelle après le bal du mardi gras, une femme le pria d'aller chercher le curé pour délivrer son mari, possédé du démon. A quelques arpents de la maison, le curé et lui aperçurent le diable au milieu d'un groupe de danseurs au-dessus de la maison de l'hôte possédé. Le diable les noya tous au matin dans le lac Simon parce qu'ils avalent dansé pendant le carême. L'hôte est mort sans être délivré pour avoir permis la danse à ses invités. Et le conteur de conclure: "Moé, j'sais ben qu'[...] c'est l'iable qui a fait c'saccage-là, l'iable en parsonne qui a été nayer ceuses qui avaient dansé su'l'carême ... Et pis vous voudriez à c'te heure que je vous laisse danser après mènuit, su' l'Mécardi des Cendre?... C'pas sacrant!"

Dans cette histoire, l'image de la fête est décrite non pas tant pour ce qu'elle est, c'est-à-dire subversive, que pour la leçon que l'on peut en tirer. L'évocation du désordre n'est pas un but en soi, mais plutôt l'occasion de renforcer les lois qu'on venait à peine de commencer à transgresser. Cette stratégie est particulièrement évidente dans la légende de Rose Latulipe, considérée comme l'une des plus populaires du folklore québécois.

\section{La Légende de Rose Latulipe ou le diable beau danseur}

Le bal du Mardi gras, qui clôt d'une façon magistrale la période des fêtes comprise entre l'Avent et le Carême, est l'expression même de la fête populaire. Aussi fait-il l'objet d'une attention particulière de la part de plusieurs auteurs. En marquant le passage abrupt du temps des réjouissances au temps des pénitences, cette fête des jours gras qu'on nomme carnaval, (moment où l'on peut encore manger des plats de viandes: carne vale), traduit l'une des principales caractéristiques de la fête, soit la rupture temporaire des activités quotidiennes. Les participants savent implicitement que la manifestation à laquelle ils participent est éphémère. Aussi entendent-ils profiter de ce bref sursis pour s'adonner à toutes sortes d'abus. Le carpe diem en accentuant le présent en souligne l'aspect aléatoire mais, en même temps, il pointe l'urgence de la jouissance. En marquant ainsi l'opposition entre deux temps antagonistes, celui du désordre pour la fête et celui de l'ordre pour la pénitence, la fête du Mardi gras se prête bien à l'exploitation du schéma transgres- 
sion-punition-réparation. Si l'Église interdit déjà les danses en temps normal, à plus forte raison en temps de pénitence. Malheur donc à ceux qui, après minuit, heure fatidique s'il en est, dansent "sur le mercredi des cendres."

Rappelons également que cette fête présente la connotation sacrificielle du carnaval étudiée par Bakhtine (1970, p. 198-276): banquet et danse qui se terminent par l'immolation d'une prétendue victime et la simulation d'une naissance, symbole de la fin de l'hiver et du nouveau du cycle végétatif que la fête de Pâques confirmera. Dans certains pays, la tradition de faire des mannequins puis de les "brûler dans la soirée au milieu d'une ronde" demeure significative à cet égard, comme l'illustre le témoignage rapporté par Anne-Marie Desdouits (1987. p. 164):

On s'attachait toujours à en faire autant que possible la représentation de quelque événement local survenu dans l'année et si d'aventure quelque fille du pays s'était laissée mettre à mal, le sujet était aussitôt trouvé et figuré avec des traits caractéristiques que la malignité locale n'avait pas de peine à interpréter.

Enfin, à la différence des autres fêtes profanes de l'année, le Mardi gras est prétexte à déguisement et mascarade. Aussitôt que venait la noirceur, les jeunes gens se déguisaient, soit en animal, en fantôme, en revenant ou en démon. Ainsi vêtus, ils faisaient la visite des maisons du village:

On se déguisait de façon à ne pas être reconnu et on ne manquait pas de manifester à chaque maison visitée. Les uns chantaient, d'autres gigeaient [...]. En retour du spectacle présenté, on recevait quelques bonbons. La tournée se poursuivait jusqu'à ce que tout le monde soit couché. Anne-Marie Desdouits (1987, p. 160).

Ces différents éléments festifs caractérisent la légende de Rose Latulipe, fort populaire au Québec - Jean Du Berger (1980), qui a étudié cette légende dans la tradition orale du Canada français, en a recueilli plus de 500 versions. On y retrouve une jeune fille victimisée, selon une violence fondatrice de paix communautaire, pour avoir dansé après minuit avec le diable qui a pris l'apparence d'un élégant jeune homme. En fait, malgré son décor socio-historique, cette légende 
d'origine populaire évoque avant tout une fête qui tourne mal. D'où son succès, largement attribuable au fait qu'elle donnait à la classe dominante des moyens de répression à l'endroit des conduites déviantes. Plusieurs écrivains l'ont fixée à l'écrit dans cette perspective, tout en la réaménageant à leur convenance.

La version littéraire la plus connue de cette légende est celle de l'écrivain Philippe Aubert de Gaspé (fils) qui parait en 1837 dans son roman l'Influence d'un livre sous le titre de "l'Étranger." Publiée au moins dix fois au XIXe siècle, cette version raconte que Rose Latulipe, une jeune fille coquette, jolie et fière, ne cesse, malgré son engagement à un jeune homme, d'encourager les attentes de ses autres soupirants. Un soir de Mardi gras, au bal traditionnel, un beau jeune homme, vêtu de velours noir, "galonné sur tous les sens," étranger à la paroisse, survient vers onze heures et demande l'hospitalité. Il invite alors la fille du propriétaire à danser; il danse divinement et Rose, envoûtée, lui accorde toutes les danses et en oublie son fiancé Gabriel Lepard. A minuit, comme c'était la coutume, le père veut arrêter la danse. Mais à la demande de l'étranger, Rose supplie son père de ne pas interrompre la fête; celui-ci cède inexplicablement à sa fille. Maitre désormais d'un espace et d'un temps que la transgression lui a cédés, l'étranger, qui n'est nul autre que le diable, poursuit hardiment sa cour auprès de sa victime qui, après une bien faible résistance, accepte de devenir son épouse bien qu'elle ne le connaisse que depuis une heure. Pendant ce temps, le curé du village, averti en songe, survient à la fête au moment où l'étranger s'apprête à emporter sa victime. Après un combat symbolique entre les forces du bien et celles du mal, le curé parvient à chasser l'adversaire qui disparait en laissant derrière lui une forte odeur de soufre. Sauvée et repentante, Rose entre alors au couvent pour expier sa faute. Cinq ans plus tard, la cloche du couvent annonce que la religieuse a krejoint son époux céleste, et une foule de curieux s'étaient réunis dans l'église, de grand matin, pour assister à ses funérailles."

Dans cette légende, toute l'affabulation est centrée sur le schème de transgression-punition. En dansant durant la période de pénitence collective qu'est le carême, Rose désobéit à un précepte religieux qui engage la communauté: le lieu de la fête devient le lieu de la faute. Aussi est-elle victimisée de façon à concourir au salut de cette même communauté. Il s'agit de rendre explicite la relation de cause à effet entre la transgression et la punition de façon à ce que tout pécheur se sente menacé des foudres divines à l'instant même de sa faute.

On aurait tort toutefois de ne s'attarder ici qu'à l'interdiction de "danser sur le mercredi des cendres," car la transgression est aussi et surtout de nature sexuelle. 
Il est bien connu que la sexualité donne lieu à toute sorte de désordre: bataille, jalousie. rixe, etc. Les bals et la danse offrent autant d'occasions de provocations, de brèches dans les cadres famillaux et sociaux bien établis. Les risques connus de la présence d'êtrangers et d'une promiscuité plus grande des femmes et des hommes, mais surtout, bien sûr, de l'excitation du moment et des ivresses car, toujours, l'alcool est généreusement distribué lors de ces fêtes, suffisent à faire éclater les mauvais mots, les injures provocatrices, les défis, voire même à faire couler le sang.

C'est dans ce sens que s'engage l'affrontement selon une dialectique triangulaire dont Rose est l'enjeu. Le conte insiste d'abord sur les causes qui désignent Rose à l'attention du diable: "c'êtait une jolie brune [...], mais elle était un peu scabreuse pour ne pas dire éventée.* De plus, "elle aimait beaucoup les divertissements.* Le conteur signale ensuite le fait que Rose *contre son ordinaire [...] avait tenu, toute la soirée, fidèle compagnie à son prétendu [Gabriel Lepard]: c'était assez naturel; ils devaient se marier à Pâques suivant." Lorsque Rose accepte l'invitation de l'étranger, "le pauvre Lepard [se mord] les lèvres à en faire sortir le sang. L'inconnu n'abandonna pas Rose du reste de la soirée, en sorte que le pauvre Gabriel, renfrogné dans son coin, ne paraissait pas manger son avoine de trop bon appétit." Puis, une "vieille et sainte" tante avertit Rose que "c'est bien mal [...] d'abandonner le bon Gabriel [...] pour ce monsieur" pas très catholique, selon elle. Mais Rose ne prête aucune attention à cet avertissement et retourne auprès de celui qui veut l'enlever de son milieu naturel. Après le coup fatal de minuit, l'identité satanique se révèle de plus en plus jusqu'à la confirmation par le curé, lequel prend la relève du fiancé dans une confrontation ultime.

Lintervention d'un membre du clergé pour délivrer la malheureuse marque de façon manifeste la récupération de l'imaginaire populaire au profit de la classe dominante. Conformément au modèle du bon chevalier délivrant la belle des griffes du dragon, le fiancé devrait normalement revenir délivrer sa bien-aimée et la légende se terminerait alors par leur mariage. Mais l'auteur opte de propos délibéré pour un dénouement qui met en valeur le rôle du curé. Celui-ci est d'abord présenté dans une pose hiératique et stéréotypée: "agenouillé depuis neuf heures du soir, [le vieux curé] ne cessait d'invoquer Dieu: le priant de pardonner les péchés que commettaient ses paroissiens dans cette nuit de désordre, le Mardi-Gras.» Un songe lui apprend le danger qui menace Rose. Il se rend en hâte au lieu du péril et engage le combat avec le diable. Dans cette confrontation, l'auteur trouve l'occasion toute désignée pour dénoncer les abus des fêtes populaires. Au curé qui lui demande la 
raison de sa présence parmi des chrétiens, le Diable réplique: "Je ne reconnais pas pour chrétiens [...] ceux qui, par mépris de votre religion, passent, à danser, à boire et à se divertir, des jours consacrés à la pénitence par vos préceptes maudits." Le curé ne se laisse pas décontenancer par ces arguments et, de son étole, il entoure Rose et la délivre des griffes du malin. "Il apparait alors comme transfiguré aux yeux de ses paroissiens:" "[...] en voyant leur vénérable pasteur qui leur avait toujours paru si timide et si faible, et maintenant si fort et courageux, face à face avec l'ennemi de Dieu et des hommes." Objet de désir dans la dialectique suprême entre le bien et le mal, Rose appartient en dernier lieu au vainqueur, le curé. Aussi doit-elle se consacrer à Dieu, qui devient alors "son époux céleste."

Au cours du XIXe siècle, plusieurs autres écrivains, dont Charles Laberge (1848), J. Ferdinand Morrissette (1883), Armand de Haerne (1886) et Charles-Marie Ducharme (1889), réaménagent la légende selon leur convenance. Mais toutes les versions évoquent une fête qui tourne mal. Véritables instruments de contrôle social et de moralisation, elles illustrent de façon exemplaire le sort réservé aux imprudentes qui aiment trop danser et s'amuser. Au coeur de chaque version, on trouve l'interdiction de danser surtout en un temps sacré. La transgression de cet interdit entraîne une punition dont le Diable est l'instrument. On constate en effet que si le personnage de la jeune fille perd à l'occasion de l'importance et que celui du prêtre disparait, le personnage du diable joue toujours un rôle prépondérant. Cet élément est significatif car il révèle l'état d'une société fortement marquée par les croyances religieuses où le diable est devenu "le symbole de la peur du péché et, par voie de conséquence, le symbole de la puissance de l'Église» (D. Mativat, 1984, p. 15). Dans une religion de peur, le diable est le principal atout de Dieu. Plus il est terrifiant, plus Dieu y gagne.

\section{Les Revenants}

Sans être aussi fréquentes que celles du diable, les interventions des revenants dans les contes canadiens-français n'en ont pas moins le même but moralisateur. D'abord elles illustrent le châtiment et les tourments réservés aux pécheurs dans l'autre monde pour les fautes qu'ils ont commises dans celui-ci. Ainsi, dans la légende d'Honoré Beaugrand (1979, p. 83-92) "Le Fantôme de l'avare," Jean-Pierre Beaudry, qui, la veille du jour de l'an, a refusê l'hospitalité à un voyageur en détresse, doit revenir après sa mort à la veille de chaque nouveau jour de l'an, 
attendre qu'un voyageur vienne frapper à sa porte. La visite du père Joseph Hervieux le sauve alors "des flammes du purgatoire où [il] brûle depuis cinquante ans, sans avoir jamais pu remplir la pénitence que Dieu [lui] avait imposée."

Les revenants interviennent également pour ramener les fêtards à l'ordre, comme en témoigne la "Légende du père Romain Chouinard," recueillie par Philippe-Joseph Aubert de Gaspé dans ses Mémoires (1866). Joséphine Lalande, une jolie jeune fille qu'on surnomme la Fine, continue à faire la coquette malgré ses fiançailles avec Hippolyte Lamonde. Pendant que celui-ci travaille au loin pour amasser le pécule nécessaire à leur mariage, elle mène une vie frivole en attirant nombre de soupirants. Un soir, vers minuit, alors qu'elle revient d'une veillée avec "une bande de jeunesse," elle aperçoit sur le perron de l'église un homme portant un surplis et un bonnet carré. Croyant qu'il s'agit du fils du bedeau qui se serait ainsi déguisé pour lui faire peur, elle fonce sur lui, vole son bonnet et l'emporte à la maison où elle le cache dans un coffre. Elle ignore cependant que ce bonnet appartient à un revenant condamné, comme son nom l'indique, à revenir sur la terre pendant trente ans pour avoir profané une leçon de catéchisme en l'absence du curé dont il avait revêtu le surplis et le bonnet. Il accomplissait justement sa dernière visite lorsque la jeune fille lui a subtilisẽ son bonnet. Au cours de la nuit, il intervient donc auprès de cette dernière pour qu'elle le lui rende. Après une nuit de frayeur, la Fine demande le lendemain à coucher dans la chambre de sa tante, mais le revenant ne lui apparaît pas moins. Dès lors, sa santé s'en trouve considérablement affectée malgré l'intervention du meilleur médecin. Elle insiste pour voir le curé à qui elle confesse sa faute. Considérant les implications surnaturelles de la situation, le pasteur organise la restitution du bonnet. Mais, au moment de le rendre, la jeune fille s'évanouit, les doigts si crispés sur le bonnet qu'il est impossible de le lui arracher. Sa santé continue donc de se détêriorer au point de mettre sa vie en danger. Revient enfin le fiancé, qui, une fois mis au courant, va rendre le bonnet et obtient du revenant un remède pour guérir la jeune fille. Le mariage peut avoir lieu une fois que Joséphine a recouvré la santé.

A l'instar de Rose Latulipe, l'intervention surnaturelle dont la Fine est victime lui sert de leçon et marque pour elle la fin de la fête. Cette fois cependant, le hẻros sotériologique n'est plus le curé, mais le fiancé. En remettant le bonnet carré au revenant, il sauve la Fine d'une mort certaine. Malgré tout, loin d'être des antagonistes, le curé et le fiancé agissent en fonction d'un même objectif: celui du retour à l'ordre. Le premier désapprouve la conduite désinvolte de certains de ses 
paroissiens, et le second, celle de sa fiancée qui refuse de se ranger. Ils parviennent à leur fin, non sans avoir d'abord reçu l'aide des forces surnaturelles.

D'autres moralia sur le respect dû aux morts, comme le montre Maurice Lemire (1993), empruntent le mythème du convive de pierre qui a été exploité en littérature par Tirso de Molina et Molière. La statue du Commandeur déifiée par Don Juan, qui l'a invité à dîner, survient effectivement pendant le repas et demande à s'asseoir à table. Dom Louis blâme alors vertement la conduite de son fils. Cette fable, qui a inspiré l'Europe, a trouvé son adaptation dans l'imaginaire québécois. Dans la version de Joseph-Charles Taché (1863, p. 166-171), intitulée "l'Hôte à Valiquet," la statue s'est muée en cadavre de meurtrier suspendu à un arbre dans une cage de fer le long de la route pour inspirer aux passants une crainte salutaire. Valiquet, qui se prépare à faire baptiser, donne un grand repas en l'honneur de son nouveau-né. En revenant de distribuer ses invitations, il passe près de la cage du pendu et, lui lançant un coup de fouet, il l'invite à son festin. Quelle n'est pas sa surprise, au moment où la fête bat son plein, de voir survenir le pendu qui demande à partager les agapes. Valiquet le supplie alors de se retirer, lui promettant service, libéra et autres prières. Le pendu n'accepte de quitter sa maison qu'après lui avoir fait promettre de venir danser au pied de sa cage le lendemain soir à minuit. Interrompues, les réjouissances laissent alors place à la récitation du "rosaire, qu'on fit suivre du De profundis." Le soir fatidique, Valiquet se rend au lieu convenu en compagnie de son fils nouvellement baptisé dans les bras. Le pendu lui dit alors: "Tu n'es pas généreux [...]. Hier soir, je me suis débarrassé de ma cage afin de pouvoir m'asseoir à ta table, et toi, cette nuit, tu viens chargé d'un fardeau afin de ne pas danser avec moi: j'avais pourtant une belle ronde à te proposer, la mesure se bat à coup de fouet." Valiquet prend alors congé de son hôte en jurant de ne jamais plus faire de telles invitations.

Cette histoire n'est pas sans rappeler celle de "la Corriveau," qu'on trouve dans Les Anciens canadiens (1863) de Philippe-Joseph Aubert de Gaspé. Pour avoir tué son mari, Josephte Corriveau a été exécutée et son cadavre, exposé à la vue des passants de la Pointe-Lévis dans une cage de fer. Le conte évoque d'abord les circonstances qui exposent François Dubé, le père du conteur José Dubé, à l'emprise de la sorcière: «il s'était diverti, comme qui dirait, à pintocher tant soit peu avec ses connaissances de la Pointe-Lévis." De plus, "il aimait un peu la goutte, [...] à telle fin qu'il portait toujours quand il voyageait, un flacon d'eau-de-vie dans son sac de loup marin." Au moment de rentrer chez lui, ses amis tentent de l'en dissuader, alléguant qu'il n'est guère prudent la nuit de "passer tout seul devant 
la cage de fer [del la Corriveau." Se targuant de bravoure, le défunt père de José décline leur invitation à coucher et se met en route. Loin de provoquer la Corriveau quand il passe près de sa cage, il lui récite un de profundi. Ces bonnes dispositions n'empêchent toutefois pas l'intervention du surnaturel, que favorise immanquablement l'heure fatale de minuit et l'habituel "flacon d'eau-de-vie." La Corriveau, qui désire se rendre au sabbat des sorciers de l'île d'Orléans et participer à la ronde infernale, cherche un chrétien pour lui faire traverser le "fleuve bénit." Elle exige du père de José qu'il la conduise, mais celui-ci se refuse à ce marché. Furieuse, elle étouffe le pauvre homme qui s'êvanouit. A son réveil, celui-ci saisit sa bouteille d'eau-de-vie pour se donner du courage et, voyant qu'elle est vide, croit que la sorcière a ingurgité la boisson.

Comme bon nombre de légendes, celle de la Corriveau met bien en évidence la régression spécifique au fonctionnement pulsionnel. Le héros dionysiaque voit dans l'extase du plaisir, où la conscience est dépossédée d'elle-même, un avantgoût de la mort, comme si celle-ci dissimulait au vivant de mystérieuses voluptés. Par son péché, ici lié à la consommation d'alcool — tantôt à la danse ou au manque de respect envers les morts, etc.-, il se met en marge du monde des vivants. Il est alors confronté à des êtres surnaturels qui l'invitent à poursuivre la fête dans le monde des morts. Tandis que le diable tente d'entraîner Rose Latulipe en enfer, que le pendu invite Valiquet à venir danser au pied de sa cage, la Corriveau demande à François Dubé de l'accompagner au sabbat de l'Ile, c'est-à-dire, pour être plus précis, le "traîner en enfer comme un hérétique." Cette expérience surnaturelle est avant tout celle d'une mort anticipée. Nouvel Énée descendu aux Enfers où il assiste à la promenade des Ombres, François Dubé est témoin de la ronde infernale des feux follets, des démons et des êtres fantastiques qui se sont donnés rendez-vous sur l'île d'Orléans. Sa "reprise de connaissance" le lendemain matin marque pour ainsi dire une résurrection initiatique, l'annonce d'un ordre renouvelé. D'où la métamorphose du héros qui sort de cette aventure diminué dans ce qui faisait sa supériorité, son courage initial. A son réveil, il a non seulement perdu souvenir de son identité: "je ne sais trop qui je suis ce matin," il est littėralement transformé comme l'avoue José: "je vous dirai en confidence que mon défunt père, qui, avant cette aventure, aurait été dans un cimetière en plein coeur de la nuit, n'était plus si hardi après cela; car il n'osait aller seul faire son train dans l'étable, après soleil couché." Le mode d'expression libidinal par excellence, la pulsion, prend dans le conte surnaturel, du fait de sa convergence avec la mort, 
son sens ultime de pulsion de mort, celle où la régression atteint son but essentiel en favorisant la renaissance.

\section{Les Loups-garous}

Après le diable et les revenants, le personnage le plus susceptible d'interrompre la fête est sans aucun doute le loup-garou. Bien qu'il soit issu du folklore, le thème du loup-garou a également des racines historiques puisqu'il y a eu dans les annales judiciaires de certains pays de véritables cas de lycanthropie, c'est-à-dire de gens qui se prenaient pour des loups et se trouvaient saisis d'une véritable folie meurtrière. Au Québec, le thème ne va pas si loin et a été essentiellement récupéré par la religion. Le loup-garou québécois, c'est d'abord un mécréant qui n'a pas fait ses pâques depuis sept ans et qui, de ce fait, est condamné la nuit à prendre la forme d'un animal. Il erre ainsi la nuit à l'aventure, seul ou avec ses semblables, et court "la galipotte" ou "court le loup-garou."

Au demeurant, le loup-garou constitue l'illustration de ce qui advient aux incorrigibles fêtards qui, en se laissant aller à leurs penchants, sont ravalés au niveau de la bête. Dans le conte "Le Feu des Roussi" de Narcisse-Henri-Édouard Faucher de Saint-Maurice, Cyprien Roussi considéré comme le rol des larons et des buveurs, n'a pas fait ses pâques depuis six ans et onze mois. Aussi les commérages vont-ils bon train dans le village, car selon les croyances populaires, après la septième année "les gens placés en aussi triste cas se transformaient en loups-garous." En fait, par sa force musculaire et ses penchants dionysiaques, Cyprien réunit dans sa personne tous les attributs qui, dans un pays où prime le travail manuel, caractérisent le héros populaire: "Il était, par droit de conquête, le roi de tous ces noceurs, roi par la verve, par ladresse, et par la force corporelle, car personne mieux que le petit Cyprien ne savait raconter une blague, adresser un coup de poing, décapuchonner avec une balle un goulot de bouteille et vider en une heure les pintes et les chopines de rhum." Mais la littérature, qui assume jusqu'à un certain point une fonction répressive dans la société, est mal placée pour rendre tẻmoignage de ce héros carnavalesque. Dans son désir d'affranchissement, le peuple, note Maurice Lemire (1986), s'est doté d'un imaginaire dominé par des dieux à son image et à sa ressemblance. C'est pourquoi la valorisation par la force physique, la prouesse et l'endurance tend à l'emporter sur le statut social. La classe dominante ne l'entend cependant pas de cette orellle. A ses yeux, 
l'exaltation de la force physique, qui fait de certains dévoyés des sortes d'idoles, est suspecte d'anarchie. Aussi, dans un imaginaire revu et corrigé, le héros populaire est-il démythifié, voire confondu pour faire prévaloir l'ordre et la loi. C'est dans cette perspective que, faute d'occulter ce personnage problématique, Faucher de SaintMaurice tente ni plus ni moins de le récupérer. Dans un premier temps, l'auteur cède la parole aux commères du village de façon à sauvegarder sa crédibilité d'homme instruit qui ne saurait cautionner la croyance populaire voulant que les chrétiens qui n'aient pas fait leurs pâques pendant sept ans soient changés en loups-garous: "Rira bien qui rira le dernier, disait dévotement la veuve Demers. Quand il sera obligé de courir les clos, et cela pendant des nuits entières, sans pouvoir se reposer, il aura le temps de songer aux remords que laissent toujours les fêtes et les impiétés." "J'ai ouĩ dire renchérit Angélique Dessaint qu'un loupgarou pouvait être un ours, chatte, chien, cheval, boeuf, crapaud. Ça dépend, paraît-il, de l'esprit malin qui lui est passé par le corps."

Malgré ce recours à une instance narrative intermédiaire, la position de Faucher de Saint-Maurice reste ambiguë. Certes il désire discréditer la conduite déviante de Cyprien, mais il ne retient pas la punition que lui fournit la croyance populaire. Il ne désavoue pas pour autant cette croyance car il va donner à son protagoniste une chance de salut en la personne de Marie, qui amène le protagoniste à s'interroger sur lui-même et à remettre en question son système axiologique. Sur les conseils de cette dernière dont il est amoureux, Cyprien fait ses pâques avant d'être changé en loup-garou. Puis, sur sa promesse de ne plus boire, Marie accepte de l'épouser. Pendant quinze ans, c'est le bonheur complet. Là où les réprimandes du clergé et la menace de la superstition ont échoué, l'amour réussit. Comme dans le mythème de la Belle et la Bête, Cyprien se métamorphose grâce à celle qu'il aime. Le mythème n'oppose cependant plus la beauté et la laideur des protagonistes, mais plutôt le caractère travailleur de l'un et l'oisiveté de l'autre. L'influence de "Marie la couturière" résulte de sa bonté - "il vous est si facile d'être bonne que je serai bon" de lui dire Cyprien, mais plus encore de son caractère travailleur, surdéterminé par le métier qu'elle exerce. "J'ai cru que le travail était la sauvegarde de tout, dira-t-elle, et je ne me suis pas trompée. J'al travaillé, et en travaillant, j'ai vu et retenu ce que les paresseux ne voient pas et le riche ne sent pas." En fait, les réprimandes de Marie sont l'occasion pour l'auteur d'interpeller ses compatriotes sur les méfaits de l'alcoolisme et de l'oisiveté.

Grâce à l'amour de Marie, Cyprien se transforme. De oisif et athée qu'il était il devient travailleur et pratiquant. Son mariage avec Marie est suivi de quinze années 
Pierre Rajotte 91

"de bonheur et d'union, quinze ans de paix, tel que Cyprien n'avait jamais osé les souhaiter lui-même à ses heures de rêverie les plus égoïstes." En principe, l'histoire devrait se terminer ainsi, mais l'auteur opte pour un dénouement malheureux. Un jour, après une journẻe de labeur, Cyprien trouve sa femme agonisante à laquelle il renouvelle sa promesse. Pendant une tempête en mer cependant, il trahit la morte et est englouti avec son fils dans les flots. Pourquoi ce dénouement malheureux? Sans doute pour répondre au schème de la transgression-punition. L'action de persuasion doit être une entreprise de contrôle et une garantie contre les défaillances possibles. A la minute même où il manque à sa promesse, Cyprien doit payer de sa vie.

Les joyeux lurons qui "courent le loup-garou" n'ont pas toujours la chance d'être sauvés in extremis par une belle. Le plus souvent, ils sont condamnés à se métamorphoser chaque nuit jusqu'à ce qu'un bon chrétien les délivre en leur redonnant une forme humaine. A cet égard, la tradition enseigne que le plus sûr moyen de les délivrer consiste à les blesser à un endroit ou l'autre du corps afin que le sang coule. Il va sans dire que ce geste est un geste de rédemption puisque le pécheur "viré loup-garou" reprend sa forme humaine et, aussitôt délivré, court se confesser et communier, tel Misaël Longneau, héros de Pamphile Lemay. Dans ce conte intitulé "Un Loup-garou," Misaël Longneau et Catherine Miquelon sont fêtês la veille de leur mariage. Au premier coup de minuit, le futur époux sort précipitamment, prétextant un malaise subit. Malgré de longues recherches, il demeure introuvable. Soudain, il réapparait, blessé à une épaule, suivi du frère de la mariée. Transformé en loup-garou, il avait tentê de s'en prendre à ce dernier qui l'avait blessé d'un coup de couteau et l'avait ainsi délivré. Les deux jeunes gens se sont engagés à garder le secret, le fiancé ayant juré de se confesser avant la cérémonie nuptiale, ce qu'il fit le lendemain.

A nouveau, la fête tourne mal. La malédiction qui pèse sur le loup-garou tient moins à sa double nature, animale et humaine, qu'au fait que ces deux natures sont dissociées et ne sont pas reconnues simultanément par la communauté. Aussi, à minuit, heure magique à laquelle les carosses se transforment en citrouilles, et les mariés en loups-garous, le fêtard doit-il quitter la fête. Le conte insiste d'ailleurs sur ce qui désigne Misaël à la transformation: "il avait rempli son verre plus d'une fois, le gaillard, car il était noceur en diable.» Le transgresseur est donc victimisé et par ce rituel est commémoré le "retour au chaos d'où doit sortir l'être régénéré," pour reprendre l'expression de Durand (1969, p. 358). En ce sens, la fête se rapproche de la grande dissolution, c'est le retour à l'état primordial qui 
est l'annonce d'un ordre renouvelé, c'est le schéma de la résurrection prochaine d'un pécheur repentant, d'un enfant prodigue "qui était mort et qui est revenu à la vie." La littérature nous donne une série d'exemples de la nécessité de la mort qui doit permettre la renaissance; il ne s'agit là que d'une reconduction d'une modalité que Durand appelle le "régime nocturne" et que Mircea Eliade formalise bien dans son Traité d'histoire des religions (1964, p. 301): "L'homme régresse provisoirement à l'état amorphe, nocturne du chaos pour pouvoir renaître avec plus de vigueur dans sa forme diurne. L'orgie, de même que l'immersion dans l'eau, annule la création mais la régénère en même temps; s'identifiant avec la totalité non différenciée précosmique, l'homme espère revenir à lui restauré et régénéré."

\section{Conclusion}

Dans son ouvrage sur Dionysos, Michel Maffesoli (1982) montre bien comment ce daimon qu'est Dionysos, s'il est l'êmissaire du monde souterrain, n'en reste pas moins celui du renouveau. Voilà bien résumé tout l'aspect contradictoire de la fête, à savoir que son aspect destructeur et subversif renvoie à une symbiose de forces, d'énergies, qui crée ou renouvelle la structuration sociale. L'excès et la frénésie de la fête commémorent l'effervescence originelle mais en même temps l'expient, l'expurgent.

Il va sans dire que pour les autorités, la meilleure façon de récupérer la fête consiste à réduire à sa plus simple expression son aspect subversif au profit de son action régénératrice. Si on interdit complètement les fêtes, on risque, comme dans la pièce les Bacchantes d'Euripide, de subir le sort de Penthée, administrateur de Thèbes, dépecé par les bacchantes parce qu'il réprouve la licence débridée et orgiaques des hommes et des femmes de sa cité. Mieux vaut alors tolérer la fête comme l'enseigne l'ancienne sagesse politique du panem et circences tout en s'efforcant de la contenir, de la maintenir décente, mais surtout de la faire tourner au profit de l'ordre.

De par sa fonction cathartique et sa fonction répressive, l'image de la fête populaire dans les contes québécois du XIXe siècle s'inscrit tout à fait dans cette perspective. Pour une part, les héros de la fête, en vivant pour les auditeurs et à leur place leur incoeurcible dêsir de dépassement, les dispensent d'y succomber eux-mêmes. Comment sans la transgression de Rose Latulipe, de Joséphine Lalande, de François Dubé, de Cyprien Roussi, de Misaël Longneau, sans ces récits 
merveilleux d'un péché, comment les fêtards ne prolongeraient-ils pas eux-mêmes leurs ébats au-delà de certaines limites. Mais cela leur devient inutile dès qu'existe le mythe, dès qu'en entendant la fable leur désir se délivre et qu'à titre d'auditeurs ils commettent eux aussi le délit. Cela fait, ils pourront ensuite rentrer tranquillement dans leur vie et contribuer au rétablissement de l'ordre. Les grands mythes ne nous apprennent-ils pas que la dérégulation est facteur d'équilibre social? La passion adultère de Tristan et Iseut, le pacte avec le diable de Faust, le désir ardent et destructeur de Don Juan, le rêve extravagant de Don Quichotte sont autant de façon de dire non à la société, de briser ponctuellement l'ordre social. Ces héros maudits, ces révoltés qui n'incarnent chacun un aspect de la condition humaine qu'à la façon d'un bouc émissaire se charge d'un péché, qui oserait douter que, s'ils vivent en nous, c'est pour nous aider à mieux nous intégrer dans le corps social? Sans eux, aucune loi ne pourrait tenir et toute vie deviendrait insupportable.

Néanmoins, force est de constater que l'image de la fête que nos auteurs exploitent est relativement peu subversive. L'évocation du chaos n'est pas un but en soi. Nulle part des nuits de Walpurgis, des sabbats ou des saturnales, aucune libation qui se prolonge en bacchanales. Le diable, ou ses avatars, apparait toujours assez tôt pour empêcher le scandale, mais on peut croire que, sans son intervention, les choses iraient beaucoup plus loin. Ainsi les lettrés ont préservé l'imaginaire populaire, l'ont fixé à l'écrit, mais non sans l'avoir d'abord émasculé de toute ambiguïté carnavalesque. Aux grands interdits bafoués du fantastique international (pratiques orgiaques, étreintes incestueuses, bestialité, union contre nature), ils substituent des problèmes de coquetterie, d'ivrognerie, d'impiété, des attitudes blasphématoires ou impudiques qui ne remettent pas grand chose en cause sinon le rigorisme de la morale catholique et le conformisme social trop étroit. "Que la fête se pénètre ou s'abâtardise de fictions religieuses, de mythes, de croyances, ne veut pas dire que ces représentations-là soient primordiales, disait Jean Duvignaud (1980, p. 26-27). Je penserais plutôt qu'elles en cachent la part maudite, évidemment dangereuse."

Les écrivains canadiens du XIXe siècle ont fondé de grands espoirs sur la littérature orale de leur pays. Ils ont prétendu la conserver tout en la mettant en valeur. A cette fin, ils ont procédé à différents types de remaniements qui répondent certes à leur volonté en quelque sorte créatrice mais aussi, et surtout, à leur souci de plier l'imagination populaire par trop subversive aux goûts esthétiques et au rigorisme moral des classes cultivées dont ils faisaient eux-mêmes partie. A la limite, le conte populaire, du moins dans la forme qu'ils retiennent, apparait comme 
un prolongement de la prédication des curés en chaire, une prédication axée sur la peur du diable et la menace du châtiment divin. Certes, il narre l'expérience de certaines limites franchies, ignorées, mais exposent aussitôt les contrevenants à en subir les conséquences. Jamais on ne danse, ne jure, ni ne boit impunément. Toute transgression avorte en cours de route et est surdéterminée par le châtiment qui frappe les transgresseurs. Et comme linterdit qui provoque la transgression, ce châtiment appartient généralement à l'imaginaire religieux: ou les héros carnavalesques sont livrés à l'emprise de Satan et ses avatars sortis tout droit de l'enfer, ou ils sont délivrés au dernier moment par un bon chrétien, ou encore par le prêtre, investi alors du rôle de héros sotériologique.

\section{Bibliographie}

Aubert de Gaspé, Philippe-Ignace-François. L'Influence d'un livre. Roman historique. Québec: Imprimé par William Cowan \& fils, 1837,122 p.

Aubert de Gaspé, Philippe-Joseph. Les Anciens Canadiens. Publié par la direction du "Foyer canadien." Québec: Desbarats et Derbishire, 1863. 411 p.

—. Mémoires. Ottawa: G.-E. Desbarats, 1866.563 p.

Bakhtine, Mikhail. L'Oeuvre de François Rabelais et la culture populaire au Moyen Age et sous la Renaissance. Paris: Gallimard, 1970. 471 p.

Beaugrand, Honoré. La Chasse-galerie [préface de François Ricard]. Montréal:

Fides, 1979. 107 p. [Première édition en 1900, Montréal, s.é., 123 p.]

Boivin, Aurélien. Le Conte littéraire québécois auXIXe siècle. Essai de bibliographie critique et analytique. Montréal: Fides, 1975. xxxviii, 385 p.

—, Le Conte fantastique québécois au XIXe siècle. Montréal: Fides, 1987. 440 p. Desdouits, Anne-Marie, La Vie traditionnelle au pays de Caux et au Canada français. Québec et Paris: Les Presses de l'Université Laval et les Éditions du CNRS, 1987. $439 \mathrm{p}$.

Du Berger, Jean. Le Diable à la danse (thèse de doctorat). Québec: Université Laval, 1980. xxxiv, $483 \mathrm{f}$.

Ducharme, Charles-Marie. Ris et Croquis. Montréal: C.O. Beauchemin \& fils, libraires-imprimeurs, 1889. $464 \mathrm{p}$.

Duquet, Édouard. Pierre et Amélie. Québec: J.-N. Duquet, libraire-éditeur, 1866 $44 \mathrm{p}$. 
Pierre Rajotte 95

Durand, Gilbert. Anthropologie structurale de l'imaginaire. Paris: Gallimard, Bibliothèque des sciences humaines, 1969. $504 \mathrm{p}$.

Duvignaud, Jean. "La Fête, aujourd'hui," dans Que la fête commence, sous la direction de Diane Pinard. Québec: La Société des festivals populaires du Québec, 1982. p. 23-27.

Eliade, Mircea. Traité d'histoire des religions. Paris: Payot, 1964. 393 p.

Faucher de Saint-Maurice, Narcisse-Henri-Édouard. "A la brunante. Contes et récits," dans L'Opinion publique, vol. III, nos 7 et 8 (15 et 22 février, 1872). p. 82; 94.

Hearne, A. de. "Le Diable au bal. Légende canadienne," dans Les Nouvelles soirées canadiennes, vol. V. Montréal: Imprimerie générale, 1886. p. 4-9.

Laberge, Charles, "Conte populaire," L'Avenir no 16 (19 février 1848), p. 1.

Larue, Hubert. "Voyage autour de l'lle d'Orléans," dans Les Soirées canadiennes. Recueil de littérature nationale, vol. I. Québec, Brousseau Frères, Éditeurs, 1861. p. 113-174.

Lemire, Maurice. "Le Discours répressif dans le conte littéraire québécois au XIXe siècle," dans Quaderni di francofonia 3, Letterature francofone del Canada. Atti del VI convegno Internazionale dell'Associazione Italiana di Studi Canadesi a Selva di Fasano (Italie), 27-31 mars 1985. Bologna: Éditrice Club, 1986.

—, La Formation de l'imaginaire littéraire au Québec, 1764-1867. Montréal: Éditions l'Hexagone, 1993 (à paraître).

Maffesoli, Michel. L'Ombre de Dionysos, contribution à une sociologie de l'orgie. Paris: Méridiens/Anthropos, Collection sociologies au quotidien, 1982. 212 p.

Mativat, Daniel "Le Fantastique québécois au XIXe siècle," Imagine, Vol. V, no 5 (juin 1984). p. 12-18.

Montigny, Louvigny Testard de. "Le Rigodon du diable. Légende du mardi gras" dans La Presse, vol. XIV, no 93 (22 fêvrier 1898), p. 5.

Morissette, Joseph-Ferdinand. "Le Diable au bal. Nouvelle," dans Au coin du feu. Nouvelles, récits et légendes. Montréal: Imprimerie Piché frères, 1883, p. 21-31.

Taché, Joseph-Charles. "Forestiers et Voyageurs," dans Les Soirées canadiennes. Recueil de littérature nationale, vol. III. Québec: Brousseau Frères, Éditeurs, 1863, p. 13-260.

Tremblay, Victor-Laurent. Au commencement était le mythe. Ottawa: Les Presses de l'Université d'Ottawa, 1991. 362 p.

Wallot, Jean-Pierre. Un Québec qui bougeait. Trame socio-politique du Québec au tournant du XIXe siècle. Montréal: Boréal Express, 1973. 345 p. 